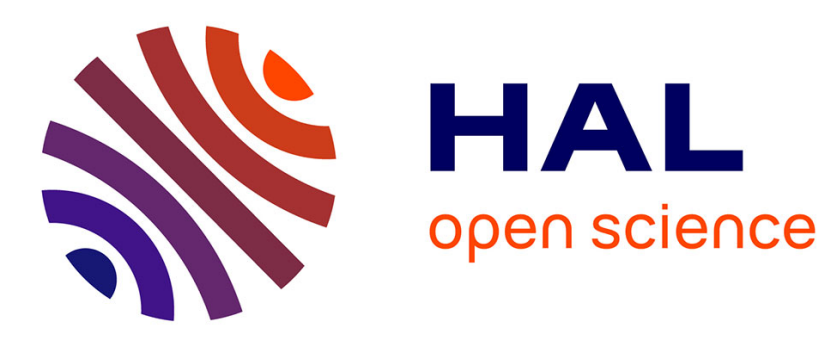

\title{
Stability of -statistics from weakly dependent observations
}

Marek Kaluszka, Andrzej Okolewski

\section{To cite this version:}

Marek Kaluszka, Andrzej Okolewski. Stability of -statistics from weakly dependent observations.

Statistics and Probability Letters, 2011, 81 (5), pp.618. 10.1016/j.spl.2010.12.015 . hal-00725106

\section{HAL Id: hal-00725106 \\ https://hal.science/hal-00725106}

Submitted on 24 Aug 2012

HAL is a multi-disciplinary open access archive for the deposit and dissemination of scientific research documents, whether they are published or not. The documents may come from teaching and research institutions in France or abroad, or from public or private research centers.
L'archive ouverte pluridisciplinaire HAL, est destinée au dépôt et à la diffusion de documents scientifiques de niveau recherche, publiés ou non, émanant des établissements d'enseignement et de recherche français ou étrangers, des laboratoires publics ou privés. 


\section{Accepted Manuscript}

Stability of $L$-statistics from weakly dependent observations

Marek Kaluszka, Andrzej Okolewski

PII:

S0167-7152(10)00361-5

DOI:

10.1016/j.spl.2010.12.015

Reference: $\quad$ STAPRO 5871

To appear in: Statistics and Probability Letters

Received date: 25 October 2010

Revised date: 20 December 2010

Accepted date: 22 December 2010

Please cite this article as: Kaluszka, M., Okolewski, A., Stability of $L$-statistics from weakly dependent observations. Statistics and Probability Letters (2011),

doi:10.1016/j.spl.2010.12.015

This is a PDF file of an unedited manuscript that has been accepted for publication. As a service to our customers we are providing this early version of the manuscript. The manuscript will undergo copyediting, typesetting, and review of the resulting proof before it is published in its final form. Please note that during the production process errors may be discovered which could affect the content, and all legal disclaimers that apply to the journal pertain. 


\title{
Stability of $L$-statistics from weakly dependent observations
}

\author{
Marek Kaluszka ${ }^{a}$, Andrzej Okolewskia, ${ }^{a, *}$ \\ ${ }^{a}$ Institute of Mathematics, Technical University of Lodz, ul. Wolczanska 215, 90-005 \\ Lodz, Poland
}

\begin{abstract}
We study the stability of moments of $L$-estimates with respect to several types of weak dependence motivated by different mixing concepts. An actuarial interpretation of the presented results is indicated.
\end{abstract}

Keywords: Order statistics; L-statistics; Spectral risk measures; Weak dependence; Mixings

\section{Introduction}

Let $X_{1}, X_{2}, \ldots, X_{n}$ be non-negative random variables defined on a common probability space $(\Omega, \mathcal{F}, \mathbf{P})$. Denote by $X_{1: n} \leq X_{2: n} \leq \ldots \leq X_{n: n}$ the order statistics from the sample $X_{1}, \ldots, X_{n}$. Linear combinations of order statistics called $L$-statistics form an important class of estimators. A review of all the developments dealing with $L$-statistics is presented in Serfling (1980) and David and Nagaraja (2003). A comprehensive survey of the current knowledge about bounds for $L$-statistics has been made by Rychlik (1998, 2001).

In financial context $L$-statistics accommodate numerous indices of economic inequality as well as risk measures of actuarial science (see Davydov et al., 2007, Greselin et al., 2009). In particular, they constitute a natural class of estimators for spectral risk measures. Asymptotic properties like

\footnotetext{
*Corresponding author; tel.: (48) 42 6313859; fax.: (48) 426363114

Email addresses: kaluszka@p.lodz.pl (Marek Kaluszka), oko@p.lodz.pl (Andrzej Okolewski)
} 
strong and weak convergence of some $L$-statistics based on independent observations to the mentioned measures were discussed e.g. by Acerbi (2002), Dowd et al. (2008) and Greselin et al. (2009). Since the assumption of mutual independence of risks is often violated in actuarial and financial practice, it becomes desirable to introduce dependence concepts which can be relevant in these sciences. Denuit et al. (2001) proved that the sum of positively dependent risks is greater in stop-loss order than the corresponding sum of independent risks. On the other hand, Darkiewicz et al. (2005) showed that there is no strict relation between values of Wang's risk measures and such indices of dependence as Pearson's, Spearmann's and Kendall's correlation coefficients. The non-asymptotic results on $L$-statistics from dependent samples (e.g. Arnold and Groeneveld, 1979, Kaluszka and Okolewski, 2001, 2005, Goroncy and Rychlik, 2008) can help to recognize some properties of (closely related to each other) empirical spectral and Wang's (1996) risk measures.

In this paper we study properties of $L$-statistics in the case when the underlying observations are weakly dependent. We consider several types of dependence motivated by mixing concepts. In Section 2 we give some evaluations on the difference of expected $L$-statistics from independent and weakly dependent samples with the same univariate marginal distributions. They are expressed in terms of numerical characteristics of independent samples. In Section 3 we present similar results on stability of variances of $L$-statistics with respect to weak dependence. The bounds can be applied for example to robust analysis of empirical spectral risk measures as well as large claims reinsurance treaties against dependence of risks (see Remark 2).

\section{Stability of expected $L$-estimates}

Let $X_{1}^{\prime}, \ldots, X_{n}^{\prime}$ be independent random variables such that $X_{i}^{\prime}={ }^{d} X_{i}$, $i=1, \ldots, n$, and let $X_{1: n}^{\prime} \leq \ldots \leq X_{n: n}^{\prime}$ be the order statistics from the sample $X_{1}^{\prime}, \ldots, X_{n}^{\prime}$. Here and subsequently, $=^{d}$ means the equality in distribution. Let $\lambda_{k}, k=1, \ldots, n$, be real numbers. Denote $L=\sum_{k=1}^{n} \lambda_{k} X_{k: n}$ and $L^{\prime}=$ $\sum_{k=1}^{n} \lambda_{k} X_{k: n}^{\prime}$. We will study the stability of $\mathbf{E} L$ with respect to dependence structures belonging to some neighbourhoods of independence motivated by mixings (for notions of $\psi$ and $\phi$ mixings see, e.g., Doukhan, 1994, p.3). They can be called weak dependencies. Let us formulate the propositions for the case of identically distributed observations, which is most frequently analysed in the literature, although the proofs provide as well the results for nonidentically distributed samples. We shall assume that the integrals appearing 
in the paper exist and are finite. Moreover, we adopt the convention that $0 / 0=0$ and set

$$
C_{i}^{k}=\left(\begin{array}{c}
i-1 \\
k
\end{array}\right)\left(\begin{array}{c}
n \\
i
\end{array}\right)
$$

Proposition 1. If there exists an $\varepsilon>0$ such that

$$
\frac{\left|\mathbf{P}\left(X_{k_{1}}>x, \ldots, X_{k_{i}}>x\right)-\mathbf{P}\left(X_{k_{1}}>x\right) \ldots \mathbf{P}\left(X_{k_{i}}>x\right)\right|}{\mathbf{P}\left(X_{k_{1}}>x\right) \ldots \mathbf{P}\left(X_{k_{i}}>x\right)} \leq \varepsilon
$$

for all $x>0$ and $1 \leq k_{1}<\ldots<k_{i} \leq n, 2 \leq i \leq n$, i.e. the $X_{i}$ 's satisfy $\psi$-mixing type 1 dependence condition, then

$$
\left|\mathbf{E}\left(L-L^{\prime}\right)\right| \leq \varepsilon \sum_{k=1}^{n}\left|\lambda_{k}\right| \sum_{i=n-k+1}^{n} C_{i}^{n-k} \mathbf{E} X_{1: i}^{\prime} .
$$

Proof. We will use the following well-known identity (cf. Blom et al., 1994, p.32)

$$
\mathbf{P}\left(\sum_{i=1}^{n} \mathbf{I}_{A_{i}} \geq k\right)=\sum_{i=k}^{n}(-1)^{i-k}\left(\begin{array}{c}
i-1 \\
k-1
\end{array}\right) \sum_{1 \leq k_{1}<\ldots<k_{i} \leq n} \mathbf{P}\left(A_{k_{1}} \cap \ldots \cap A_{k_{i}}\right),
$$

where $A_{1}, \ldots, A_{n}$ are arbitrary events and $\mathbf{I}_{A}$ denotes the indicator of $A$. Of course

$$
\mathbf{P}\left(X_{k: n} \leq x\right)=1-\mathbf{P}\left(\sum_{i=1}^{n} \mathbf{I}\left(X_{i}>x\right) \geq n-k+1\right) .
$$

From (3) we have

$$
\mathbf{P}\left(X_{k: n}>x\right)=\sum_{i=n-k+1}^{n}(-1)^{i-(n-k+1)}\left(\begin{array}{c}
i-1 \\
n-k
\end{array}\right) \sum_{1 \leq k_{1}<\ldots<k_{i} \leq n} \mathbf{P}\left(X_{k_{1}}>x, \ldots, X_{k_{i}}>x\right) .
$$

Combining (4) with the analogous formula for $X^{\prime}$ s leads to

$$
\begin{gathered}
\mathbf{E}\left(L-L^{\prime}\right)=\sum_{k=1}^{n} \lambda_{k} \sum_{i=n-k+1}^{n}(-1)^{i-(n-k+1)}\left(\begin{array}{c}
i-1 \\
n-k
\end{array}\right) \int_{0}^{\infty} \sum_{1 \leq k_{1}<\ldots<k_{i} \leq n}\left[\mathbf { P } \left(X_{k_{1}}>x,\right.\right. \\
\left.\left.\ldots, X_{k_{i}}>x\right)-\mathbf{P}\left(X_{k_{1}}^{\prime}>x\right) \ldots \mathbf{P}\left(X_{k_{i}}^{\prime}>x\right)\right] d x .
\end{gathered}
$$


Hence, by (1),

$$
\left|\mathbf{E}\left(L-L^{\prime}\right)\right| \leq \varepsilon \sum_{k=1}^{n}\left|\lambda_{k}\right| \sum_{i=n-k+1}^{n}\left(\begin{array}{c}
i-1 \\
n-k
\end{array}\right) \sum_{1 \leq k_{1}<\ldots<k_{i} \leq n} \mathbf{E} X_{1: k_{1}, \ldots, k_{i}}^{\prime},
$$

where $X_{j: k_{1}, \ldots, k_{i}}^{\prime}$ denotes the $j$ th order statistics from the sample $X_{k_{1}}^{\prime}, \ldots, X_{k_{i}}^{\prime}$. For identically distributed observations (6) takes the form (2).

Proposition 2. Suppose that the $X_{i}$ 's are $\psi$-mixing type 2 dependent, that is, there exists an $\varepsilon>0$ such that

$$
\frac{\left|\mathbf{P}\left(X_{k_{1}} \leq x, \ldots, X_{k_{i}} \leq x\right)-\mathbf{P}\left(X_{k_{1}} \leq x\right) \ldots \mathbf{P}\left(X_{k_{i}} \leq x\right)\right|}{1-\mathbf{P}\left(X_{k_{1}} \leq x\right) \ldots \mathbf{P}\left(X_{k_{i}} \leq x\right)} \leq \varepsilon
$$

for any $x>0$ and all $1 \leq k_{1}<\ldots<k_{i} \leq n, 2 \leq i \leq n$. Then

$$
\left|\mathbf{E}\left(L-L^{\prime}\right)\right| \leq \varepsilon \sum_{i=1}^{n}\left(\sum_{k=1}^{i} C_{i}^{k-1}\left|\lambda_{k}\right|\right) \mathbf{E} X_{i: i}^{\prime} .
$$

Proof. From David and Nagaraja (2003, p. 99) we have

$\mathbf{E} L=\sum_{k=1}^{n} \lambda_{k} \int_{0}^{\infty}\left(1-\sum_{i=k}^{n}(-1)^{i-k}\left(\begin{array}{c}i-1 \\ k-1\end{array}\right) \sum_{1 \leq k_{1}<\ldots<k_{i} \leq n} \mathbf{P}\left(X_{k_{1}} \leq x, \ldots, X_{k_{i}} \leq x\right)\right) d x$.

Hence

$\mathbf{E}\left(L-L^{\prime}\right)=\int_{0}^{\infty} \sum_{k=1}^{n} \lambda_{k} \sum_{i=k}^{n}(-1)^{i-k}\left(\begin{array}{c}i-1 \\ k-1\end{array}\right) \sum_{\substack{1 \leq k_{1}<\ldots<k_{i} \leq n \\-}}\left[\mathbf{P}\left(X_{k_{1}} \leq x\right) \ldots \mathbf{P}\left(X_{k_{i}} \leq x\right)\right.$

and, by (7),

$$
\begin{aligned}
\left|\mathbf{E}\left(L-L^{\prime}\right)\right| & \leq \varepsilon \sum_{k=1}^{n}\left|\lambda_{k}\right| \sum_{i=k}^{n}\left(\begin{array}{c}
i-1 \\
k-1
\end{array}\right) \sum_{1 \leq k_{1}<\ldots<k_{i} \leq n} \int_{0}^{\infty}\left[1-\mathbf{P}\left(X_{i: k_{1} \ldots k_{i}}^{\prime} \leq x\right)\right] d x \\
& =\varepsilon \sum_{k=1}^{n}\left|\lambda_{k}\right| \sum_{i=k}^{n}\left(\begin{array}{c}
i-1 \\
k-1
\end{array}\right) \sum_{1 \leq k_{1}<\ldots<k_{i} \leq n} \mathbf{E} X_{i: k_{1}, \ldots, k_{i}}^{\prime}
\end{aligned}
$$

which gives (8). 
Proposition 3. Assume that for an $\varepsilon>0$ and all $1 \leq k_{1}<\ldots<k_{i} \leq n$, $2 \leq i \leq n$,

$$
\sup _{x>0}\left|\mathbf{P}\left(X_{k_{i}}>x \mid X_{k_{i-1}}>x, \ldots, X_{k_{1}}>x\right)-\mathbf{P}\left(X_{k_{i}}>x\right)\right| \leq \varepsilon
$$

i.e. the $X_{i}$ 's are $\phi$-mixing type dependent. Then

$$
\left|\mathbf{E}\left(L-L^{\prime}\right)\right| \leq \varepsilon \sum_{k=1}^{n}\left|\lambda_{k}\right| \sum_{i=n-k+1}^{n} C_{i}^{n-k} \sum_{j=2}^{i} \sum_{s=0}^{i-j}\left(\begin{array}{c}
i-j \\
s
\end{array}\right) \varepsilon^{i-j-s} \mathbf{E} X_{1: j+s-1}^{\prime} .
$$

Proof. From (5) we have

$$
\begin{aligned}
\mathbf{E}\left(L-L^{\prime}\right)= & \sum_{k=1}^{n} \lambda_{k} \sum_{i=n-k+1}^{n}(-1)^{i-(n-k+1)}\left(\begin{array}{c}
i-1 \\
n-k
\end{array}\right) \\
& \int_{0}^{\infty} \sum_{1 \leq k_{1}<\ldots<k_{i} \leq n}\left[\prod_{j=1}^{i} a_{j}(x)-\prod_{j=1}^{i} b_{j}(x)\right] d x,
\end{aligned}
$$

where $a_{1}(x)=\mathbf{P}\left(X_{k_{1}}>x\right), a_{j}(x)=\mathbf{P}\left(X_{k_{j}}>x \mid X_{k_{j-1}}>x, \ldots, X_{k_{1}}>x\right)$ for $2 \leq j \leq n$ and $b_{j}(x)=\mathbf{P}\left(X_{k_{j}}^{\prime}>x\right)$ for $1 \leq j \leq n$. Applying the identity

$$
\prod_{j=1}^{i} a_{j}-\prod_{j=1}^{i} b_{j}=\sum_{j=1}^{i}\left(a_{j}-b_{j}\right)\left[\prod_{r=1}^{j-1} b_{r}\right]\left[\prod_{r=j+1}^{i} a_{r}\right]
$$

with $\prod_{r=s}^{t} c_{r}=1$ for $s>t$, we get

$$
\begin{aligned}
\left|\mathbf{E}\left(L-L^{\prime}\right)\right| \leq \varepsilon \sum_{k=1}^{n}\left|\lambda_{k}\right| \sum_{i=n-k+1}^{n}\left(\begin{array}{c}
i-1 \\
n-k
\end{array}\right) \sum_{1 \leq k_{1}<\ldots<k_{i} \leq n} \\
\sum_{j=2}^{i} \int_{0}^{\infty}\left[\prod_{r=1}^{j-1} b_{r}(x)\right]\left[\prod_{r=j+1}^{i}\left(\varepsilon+b_{r}(x)\right)\right] d x,
\end{aligned}
$$

which for identically distributed observations leads to (11).

Next result concerns the dependence type motivated by asymptotic quadrant independence introduced by Birkel (1992). 
Proposition 4. Let

$$
\sup _{x>0} \frac{\left|\mathbf{P}\left(X_{k_{1}}>x, \ldots, X_{k_{i}}>x\right)-\mathbf{P}\left(X_{k_{1}}>x\right) \ldots \mathbf{P}\left(X_{k_{i}}>x\right)\right|}{\mathbf{P}\left(X_{k_{1}}>x\right) \mathbf{P}\left(X_{k_{1}} \leq x\right) \ldots \mathbf{P}\left(X_{k_{i}}>x\right) \mathbf{P}\left(X_{k_{i}} \leq x\right)} \leq \varepsilon
$$

for an $\varepsilon>0$ and all $1 \leq k_{1}<\ldots<k_{i} \leq n, 2 \leq i \leq n$. Then

$$
\left|\mathbf{E}\left(L-L^{\prime}\right)\right| \leq \varepsilon \sum_{k=1}^{n}\left|\lambda_{k}\right| \sum_{i=k}^{n} C_{i}^{k-1} \mathbf{E}\left(X_{1: i}^{\prime \prime}-X_{i: i}^{\prime}\right)_{+},
$$

where $x_{+}=\max \{0, x\}$ and $X_{1}^{\prime}, \ldots, X_{n}^{\prime}$ and $X_{1}^{\prime \prime}, \ldots, X_{n}^{\prime \prime}$ are independent samples such that $X_{i}^{\prime}={ }^{d} X_{i}^{\prime \prime}={ }^{d} X_{i}$ for $i=1, \ldots, n$.

Proof. By (5), (13) and Fubini's theorem, we obtain

$$
\begin{aligned}
\left|\mathbf{E}\left(L-L^{\prime}\right)\right| \leq \varepsilon & \sum_{k=1}^{n}\left|\lambda_{k}\right| \sum_{i=n-k+1}^{n}\left(\begin{array}{c}
i-1 \\
n-k
\end{array}\right) \int_{0}^{\infty} \sum_{1 \leq k_{1}<\ldots<k_{i} \leq n} \\
& \prod_{j=1}^{i} \mathbf{P}\left(X_{k_{j}} \leq x\right) \mathbf{P}\left(X_{k_{j}}>x\right) d x \\
= & \varepsilon \sum_{k=1}^{n}\left|\lambda_{k}\right| \sum_{i=n-k+1}^{n}\left(\begin{array}{c}
i-1 \\
n-k
\end{array}\right) \sum_{1 \leq k_{1}<\ldots<k_{i} \leq n} \\
\mathbf{E} \int_{0}^{\infty} \mathbf{I}\left(X_{i: k_{1}, \ldots, k_{i}}^{\prime} \leq x\right) \mathbf{I}\left(X_{1: k_{1}, \ldots, k_{i}}^{\prime \prime}>x\right) d x & \mathbf{E}\left(X_{1: k_{1}, \ldots, k_{i}}^{\prime \prime}-X_{i: k_{1}, \ldots, k_{i}}^{\prime}\right)_{+}
\end{aligned}
$$

and this implies (14).

Example 1. Let $X_{1}, \ldots, X_{n}$ have the standard exponential distribution. Then $\mathbf{E} X_{k: n}^{\prime}=\sum_{i=n-k+1}^{n} 1 / i$ (see David and Nagaraja, 2003, p. 52), and consequently the bounds of Propositions 1,2,3 and 4, respectively, take the forms

$$
\begin{aligned}
& \left|\mathbf{E}\left(X_{k: n}-X_{k: n}^{\prime}\right)\right| \leq \varepsilon \sum_{i=n-k+1}^{n} C_{i}^{n-k} \frac{1}{i}, \\
& \left|\mathbf{E}\left(X_{k: n}-X_{k: n}^{\prime}\right)\right| \leq \varepsilon \sum_{i=k}^{n} C_{i}^{k-1} \sum_{j=1}^{i} \frac{1}{j},
\end{aligned}
$$




$$
\left|\mathbf{E}\left(X_{k: n}-X_{k: n}^{\prime}\right)\right| \leq \varepsilon \sum_{i=n-k+1}^{n} C_{i}^{n-k} \sum_{j=2}^{i} \sum_{s=0}^{i-j}\left(\begin{array}{c}
i-j \\
s
\end{array}\right) \frac{\varepsilon^{i-j-s}}{j+s-1}
$$

and

$$
\left|\mathbf{E}\left(X_{k: n}-X_{k: n}^{\prime}\right)\right| \leq \varepsilon \sum_{i=k}^{n} C_{i}^{k-1}\left[i\left(\begin{array}{c}
2 i \\
i
\end{array}\right)\right]^{-1} .
$$

Remark 1. The formulae (6), (9), (12) and (15) provide the bounds for the case when the weakly dependent observations $X_{1}, \ldots, X_{n}$ are non-identically distributed.

Dependence neighbourhoods (1), (10) and (13) for $n=2$ contain some important classes of copulas. For example, the Farlie-Gumbel-Morgenstern, Ali-Mikhail-Haq and Plackett families of copulas (see Matuła, 2005) with parameters from the intervals $[0, \varepsilon],[0, \varepsilon /(1+\varepsilon)]$ and $[1,1+\varepsilon)$, respectively, belong to these neighbourhoods.

Instead of using Kolmogorov-type neighbourhoods of independence, one can consider the other types of neighbourhoods. Let us give one such example for the case of $n=2$, which is intensively studied in reliability theory (see e.g. Balakrishnan and Lai, 2009). We have

$$
\mathbf{E}\left(L-L^{\prime}\right)=\left(\lambda_{1}-\lambda_{2}\right)\left(\mathbf{E} X_{1: 2}-\mathbf{E} X_{1: 2}^{\prime}\right)=\left(\lambda_{2}-\lambda_{1}\right)\left(\mathbf{E} X_{2: 2}-\mathbf{E} X_{2: 2}^{\prime}\right),
$$

since $X_{2: 2}=X_{1}+X_{2}-X_{1: 2}$. Obviously,

$$
\mathbf{E} X_{2: 2}-\mathbf{E} X_{2: 2}^{\prime}=\int_{0}^{\infty}\left[\mathbf{P}\left(X_{2: 2}^{\prime} \leq t\right)-\mathbf{P}\left(X_{2: 2} \leq t\right)\right] d t .
$$

If $F_{1}=F_{2}=F$, then

$$
\left|\mathbf{E}\left(L-L^{\prime}\right)\right| \leq\left|\lambda_{2}-\lambda_{1}\right| \int_{0}^{1}\left|C(t, t)-t^{2}\right| d t,
$$

where $C(t, t)=\mathbf{P}\left(X_{1} \leq F^{-1}(t), X_{2} \leq F^{-1}(t)\right)$. In the case when $C$ is a generalized Farlie-Gumbel-Morgenstern copula, i.e.

$$
C(x, y)=x y+\rho x^{b} y^{b}(1-x)^{a}(1-y)^{a}, \quad a, b \geq 1,|\rho| \leq 1,
$$

we obtain

$$
\left|\mathbf{E}\left(L-L^{\prime}\right)\right| \leq|\rho|\left|\lambda_{2}-\lambda_{1}\right| B(2 a+1,2 b+1),
$$

where $B(x, y)$ denotes the Euler beta function. In particular, if $|\rho| \leq \varepsilon$ for some $\varepsilon$, then $\left|\mathbf{E}\left(L-L^{\prime}\right)\right| \leq \varepsilon\left|\lambda_{2}-\lambda_{1}\right| / 3$ for arbitrary $a, b \geq 1$, since $\int_{0}^{1} x^{2 a}(1-x)^{2 b} d x \leq \min \{1 /(2 a+1), 1 /(2 b+1)\}$. 
Remark 2. The presented inequalities can be applied to evaluate the weak dependent insurance risks. Suppose that $X_{1}, \ldots, X_{n}$ is a homogeneous portfolio of risks satisfying $\psi$-mixing condition (7). If an insurer is able to determine the parameter $\varepsilon$ for this mixing type dependence model and if he is willing to use some empirical spectral risk measure $\sum_{i=1}^{n} \lambda_{i} X_{i: n}$ with some $0 \leq \lambda_{1} \leq \ldots \leq \lambda_{n}$, then the bound (8) says that the additional (in comparison to the case of independent risks) loading related to the dependence of risks cannot exceed the coefficient determined by independent risks multiplied by $\varepsilon$. If the dependence is really weak, i.e. $\varepsilon$ is close to zero, then the loading should be small. Another application of $L$-statistics to insurance is the large claim reinsurance treaty (cf. Kremer, 1998). For this reinsurance contract not only the net premium but also the risk measured by variance may be of interest.

\section{Stability of second moments of $L$-statistics}

We will restrict our attention to one mixing type of dependence. The other mixings may be treated analogously. Let us examine first the variances of single order statistics.

Proposition 5. Assume that there exists an $\varepsilon>0$ such that inequality (1) is satisfied for all $x>0$ and all $1 \leq k_{1}<\ldots<k_{i} \leq n, 2 \leq i \leq n$. Then for any $k$

$$
\begin{aligned}
\left|\operatorname{Var} X_{k: n}-\operatorname{Var} X_{k: n}^{\prime}\right| & \leq \varepsilon \sum_{i=n-k+1}^{n} C_{i}^{n-k}\left(\mathbf{E}\left[X_{1: i}^{\prime}\right]^{2}+2 \mathbf{E} X_{1: i}^{\prime} \mathbf{E} X_{k: n}^{\prime}\right) \\
& +\varepsilon^{2}\left[\sum_{i=n-k+1}^{n} C_{i}^{n-k} \mathbf{E} X_{1: i}^{\prime}\right]^{2} .
\end{aligned}
$$

Proof. From Hoeffding's identity (see, e.g., Szekli, 1995, p. 136) for nonnegative random variables

$$
\operatorname{Var} X=\int_{\mathbf{R}_{+}^{2}}[\mathbf{P}(X>x \vee y)-\mathbf{P}(X>x) \mathbf{P}(X>y)] d x d y,
$$

we obtain

$\operatorname{Var} X_{k: n}-\operatorname{Var} X_{k: n}^{\prime}=\int_{\mathbf{R}_{+}^{2}}\left[\mathbf{P}\left(X_{k: n}>x \vee y\right)-\mathbf{P}\left(X_{k: n}^{\prime}>x \vee y\right)\right] d x d y$ 


$$
\begin{aligned}
& +\int_{\mathbf{R}_{+}^{2}} \mathbf{P}\left(X_{k: n}^{\prime}>y\right)\left[\mathbf{P}\left(X_{k: n}^{\prime}>x\right)-\mathbf{P}\left(X_{k: n}>x\right)\right] d x d y \\
& +\int_{\mathbf{R}_{+}^{2}}\left[\mathbf{P}\left(X_{k: n}>x\right)-\mathbf{P}\left(X_{k: n}^{\prime}>x\right)\right]\left[\mathbf{P}\left(X_{k: n}^{\prime}>y\right)-\mathbf{P}\left(X_{k: n}>y\right)\right] d x d y \\
& +\int_{\mathbf{R}_{+}^{2}} \mathbf{P}\left(X_{k: n}^{\prime}>x\right)\left[\mathbf{P}\left(X_{k: n}^{\prime}>y\right)-\mathbf{P}\left(X_{k: n}>y\right)\right] d x d y
\end{aligned}
$$

where $x \vee y=\max \{x, y\}$. By (4) and (1),

$$
\begin{aligned}
& \left|\int_{0}^{\infty}\left[\mathbf{P}\left(X_{k: n}>y\right)-\mathbf{P}\left(X_{k: n}^{\prime}>y\right)\right] d y\right| \\
& \quad \leq \varepsilon \sum_{i=n-k+1}^{n}\left(\begin{array}{c}
i-1 \\
n-k
\end{array}\right) \sum_{1 \leq k_{1}<\ldots<k_{i} \leq n} \int_{0}^{\infty} \mathbf{P}\left(X_{k_{1}, \ldots, k_{i}}^{\prime}>y\right) d y .
\end{aligned}
$$

Combining (18) and (19) we get

$$
\begin{aligned}
\left|\operatorname{Var} X_{k: n}-\operatorname{Var} X_{k: n}^{\prime}\right| & \leq 2 \varepsilon \sum_{i=n-k+1}^{n}\left(\begin{array}{c}
i-1 \\
n-k
\end{array}\right) \sum_{1 \leq k_{1}<\ldots<k_{i} \leq n} \int_{0}^{\infty} \int_{y}^{\infty} \mathbf{P}\left(X_{1: k_{1}, \ldots, k_{i}}^{\prime}>x\right) d x d y \\
& +2 \varepsilon \mathbf{E} X_{k: n}^{\prime} \sum_{i=n-k+1}^{n}\left(\begin{array}{c}
i-1 \\
n-k
\end{array}\right) \sum_{1 \leq k_{1}<\ldots<k_{i} \leq n} \mathbf{E} X_{1: k_{1}, \ldots, k_{i}}^{\prime} \\
& +\varepsilon^{2}\left[\sum_{i=n-k+1}^{n}\left(\begin{array}{c}
i-1 \\
n-k
\end{array}\right) \sum_{1 \leq k_{1}<\ldots<k_{i} \leq n} \mathbf{E} X_{1: k_{1}, \ldots, k_{i}}^{\prime}\right]^{2} .
\end{aligned}
$$

Applying the identity: $2 \int_{0}^{\infty} \int_{y}^{\infty} \mathbf{P}(X>x) d x d y=\mathbf{E} X^{2}$, leads to (17).

Under a stronger mixing condition, the result of Proposition 5 can be extended to $L$-statistics.

Proposition 6. Suppose that there is an $\varepsilon>0$ such that

$$
\sup _{x, y>0}\left|\frac{\mathbf{P}\left(X_{k_{1}}>x_{1}, \ldots, X_{k_{i}}>x_{i}\right)}{\mathbf{P}\left(X_{k_{1}}>x_{1}\right) \ldots \mathbf{P}\left(X_{k_{i}}>x_{i}\right)}-1\right| \leq \varepsilon,
$$

where $x_{j}=x$ or $x_{j}=y, j=1, \ldots, i, 1 \leq k_{1}<\ldots<k_{i} \leq n$ and $2 \leq i \leq n$.

Then

(i) for $k<l$

$$
\left|\operatorname{Cov}\left(X_{k: n}, X_{l: n}\right)-\operatorname{Cov}\left(X_{k: n}^{\prime}, X_{l: n}^{\prime}\right)\right| \leq \varepsilon B_{k l},
$$


in which

$$
B_{k l}=w_{l} \mathbf{E} X_{k: n}^{\prime}+w_{k} \mathbf{E} X_{l: n}^{\prime}+\frac{1}{2}\left(v_{k}+v_{k l}\right)+\varepsilon w_{k} w_{l}
$$

where $w_{k}, v_{k}$ and $v_{k l}$ are defined below by (24), (27) and (31), respectively; (ii)

$$
\left|\operatorname{Var} L-\operatorname{Var} L^{\prime}\right| \leq \varepsilon\left[\sum_{k=1}^{n} \lambda_{k}^{2} B_{k k}+2 \sum_{k=1}^{n-1} \sum_{l=k+1}^{n}\left|\lambda_{k} \lambda_{l}\right| B_{k l}\right]
$$

with $B_{k k}=2 w_{k} \mathbf{E} X_{k: n}^{\prime}+v_{k}+\varepsilon w_{k}^{2}$.

Proof. By Hoeffding's identity,

$$
\operatorname{Cov}\left(X_{k: n}, X_{l: n}\right)-\operatorname{Cov}\left(X_{k: n}^{\prime}, X_{l: n}^{\prime}\right)=I_{1}+I_{2}+I_{3}+I_{4},
$$

where

$$
\begin{aligned}
& I_{1}=\int_{\mathbf{R}_{+}^{2}}\left[\mathbf{P}\left(X_{k: n}>x, X_{l: n}>y\right)-\mathbf{P}\left(X_{k: n}^{\prime}>x, X_{l: n}^{\prime}>y\right)\right] d x d y, \\
& I_{2}=\int_{\mathbf{R}_{+}^{2}} \mathbf{P}\left(X_{k: n}^{\prime}>x\right)\left[\mathbf{P}\left(X_{l: n}^{\prime}>y\right)-\mathbf{P}\left(X_{l: n}>y\right)\right] d x d y, \\
& I_{3}=\int_{\mathbf{R}_{+}^{2}} \mathbf{P}\left(X_{l: n}^{\prime}>y\right)\left[\mathbf{P}\left(X_{k: n}^{\prime}>x\right)-\mathbf{P}\left(X_{k: n}>x\right)\right] d x d y, \\
& I_{4}=\int_{\mathbf{R}_{+}^{2}}\left[\mathbf{P}\left(X_{l: n}>y\right)-\mathbf{P}\left(X_{l: n}^{\prime}>y\right)\right]\left[\mathbf{P}\left(X_{k: n}^{\prime}>x\right)-\mathbf{P}\left(X_{k: n}>x\right)\right] d x d y .
\end{aligned}
$$

Let us evaluate each $I_{j}, j=1, \ldots, 4$, separately. Proceeding analogously as in the proof of Proposition 5 we obtain

$$
\left|I_{2}\right| \leq \varepsilon \mathbf{E} X_{k: n}^{\prime} w_{l}, \quad\left|I_{3}\right| \leq \varepsilon \mathbf{E} X_{l: n}^{\prime} w_{k} \quad \text { and } \quad\left|I_{4}\right| \leq \varepsilon^{2} w_{k} w_{l}
$$

in which

$$
w_{j}=\sum_{i=n-j+1}^{n}\left(\begin{array}{c}
i-1 \\
n-j
\end{array}\right) \sum_{1 \leq k_{1}<\ldots<k_{i} \leq n} \mathbf{E} X_{1: k_{1}, \ldots, k_{i}}^{\prime} .
$$


Observe that

$$
\begin{aligned}
I_{1}= & \int_{0}^{\infty} \int_{0}^{y}\left[\mathbf{P}\left(X_{k: n}>x, X_{l: n}>y\right)-\mathbf{P}\left(X_{k: n}^{\prime}>x, X_{l: n}^{\prime}>y\right)\right] d x d y \\
& +\int_{0}^{\infty} \int_{y}^{\infty}\left[\mathbf{P}\left(X_{k: n}>x\right)-\mathbf{P}\left(X_{k: n}^{\prime}>x\right)\right] d x d y \equiv I_{11}+I_{12} .
\end{aligned}
$$

Applying formula (4) to the second integral of (25) gives

$$
\begin{aligned}
\left|I_{12}\right| & \leq \varepsilon \sum_{i=n-k+1}^{n}\left(\begin{array}{c}
i-1 \\
n-k
\end{array}\right) \sum_{1 \leq k_{1}<\ldots<k_{i} \leq n} \int_{0}^{\infty} \int_{y}^{\infty} \mathbf{P}\left(X_{k_{1}}^{\prime}>x, \ldots, X_{k_{i}}^{\prime}>x\right) d x d y \\
& =\varepsilon v_{k} / 2,
\end{aligned}
$$

where

$$
v_{k}=\sum_{i=n-k+1}^{n}\left(\begin{array}{c}
i-1 \\
n-k
\end{array}\right) \sum_{1 \leq k_{1}<\ldots<k_{i} \leq n} \mathbf{E}\left[X_{1: k_{1}, \ldots, k_{i}}^{\prime}\right]^{2} .
$$

In order to evaluate the first integral $I_{11}$ of (25) we use the identity

$$
\mathbf{I}\left(x_{k: n}>x\right)=\sum_{i=n-k+1}^{n}(-1)^{i-(n-k+1)}\left(\begin{array}{c}
i-1 \\
n-k
\end{array}\right) \sum_{1 \leq k_{1}<\ldots<k_{i} \leq n} \mathbf{I}\left(x_{k_{1}}>x, \ldots, x_{k_{i}}>x\right),
$$

which can be deduced from (4) by putting $X_{k}=x_{k}$ for $k=1, \ldots, n$. It follows that

$$
\begin{aligned}
\mathbf{P}\left(X_{k: n}>x, X_{l: n}>y\right) & =\mathbf{E} \sum_{i=n-k+1}^{n} \sum_{j=n-l+1}^{n} a_{i j} \sum_{1 \leq k_{1}<\ldots<k_{i} \leq n} \\
& \sum_{1 \leq l_{1}<\ldots<l_{j} \leq n} \mathbf{I}\left(X_{k_{1}}>x, \ldots, X_{k_{i}}>x, X_{l_{1}}>y, \ldots, X_{l_{j}}>y\right)
\end{aligned}
$$

with

$$
a_{i j}=(-1)^{i+j-(n-k+1)-(n-l+1)}\left(\begin{array}{c}
i-1 \\
n-k
\end{array}\right)\left(\begin{array}{l}
j-1 \\
n-l
\end{array}\right) .
$$

If $k_{s}=l_{z}$ for some $s, z$, then $\mathbf{I}\left(X_{k_{s}}>x, X_{l_{z}}>y\right)=\mathbf{I}\left(X_{k_{s}}>y\right)$ for $x<y$ (cf. the definition of $I_{11}$ in (25)). Therefore

$$
\left|\mathbf{P}\left(X_{k: n}>x, X_{l: n}>y\right)-\mathbf{P}\left(X_{k: n}^{\prime}>x, X_{l: n}^{\prime}>y\right)\right|
$$




$$
\begin{aligned}
\leq & \varepsilon \sum_{i=n-k+1}^{n} \sum_{j=n-l+1}^{n}\left|a_{i j}\right| \sum_{1 \leq k_{1}<\ldots<k_{i} \leq n} \sum_{1 \leq l_{1}<\ldots<l_{j} \leq n} \\
& \mathbf{P}\left(\bigcap_{s \in S_{i j}}\left\{X_{s}^{\prime}>x\right\} \cap \bigcap_{z \in Z_{j}}\left\{X_{z}^{\prime}>y\right\}\right),
\end{aligned}
$$

where $Z_{j}=\left\{l_{1}, \ldots, l_{j}\right\}$ and $S_{i j}=\left\{k_{1}, \ldots, k_{i}\right\} \backslash Z_{j}$. Since

$$
\begin{array}{rl}
\int_{0}^{\infty} \int_{0}^{y} & \mathbf{P}\left(\bigcap_{s \in S_{i j}}\left\{X_{s}^{\prime}>x\right\} \cap \bigcap_{z \in Z_{j}}\left\{X_{z}^{\prime}>y\right\}\right) d x d y \\
& =\mathbf{E} \int_{0}^{\infty} \mathbf{I}\left(X_{1: Z_{j}}^{\prime}>y\right) \int_{0}^{y} \mathbf{I}\left(X_{1: S_{i j}}^{\prime}>x\right) d x d y \\
& =\mathbf{E} \int_{0}^{\infty} \mathbf{I}\left(X_{1: Z_{j}}^{\prime}>y\right) \min \left(y, X_{1: S_{i j}}^{\prime}\right) d y \\
& =\int_{0}^{\infty} \mathbf{P}\left(X_{1: Z_{j}}^{\prime}>y\right) \mathbf{E} \min \left(y, X_{1: S_{i j}}^{\prime}\right) d y \\
& \leq \int_{0}^{\infty} y \mathbf{P}\left(X_{1: Z_{j}}^{\prime}>y\right) d y=\frac{1}{2} \mathbf{E}\left[X_{1: Z_{j}}^{\prime}\right]^{2} \leq \frac{1}{2} \mathbf{E}\left[X_{n: n}^{\prime}\right]^{2}<\infty,
\end{array}
$$

we have, by (25), (28) and (29), that

$$
\left|I_{11}\right| \leq \varepsilon v_{k l} / 2
$$

with

$$
v_{k l}=\sum_{i=n-k+1}^{n}\left(\begin{array}{l}
n \\
i
\end{array}\right) \sum_{j=n-l+1}^{n}\left|a_{i j}\right| \sum_{1 \leq l_{1}<\ldots<l_{j} \leq n} \mathbf{E}\left[X_{1: Z_{j}}^{\prime}\right]^{2},
$$

where $X_{1: A}^{\prime}$ is the minimal order statistics from the sample of all the $X_{i}^{\prime}$ s which indices are in the set $A$. Combining (30) with (26), (25) and (23) leads to (i). The part (ii) is a straightforward consequence of (i) and the last formula in the proof of Proposition 5.

Example 2. If $X_{1}, \ldots, X_{n}$ have the standard exponential distribution, then the coefficients in the definitions of $B_{k k}$ and $B_{k l}$ in Proposition 6 take the forms $w_{k}=\sum_{i=n-k+1}^{n} C_{i}^{n-k} / i, v_{k}=2 \sum_{i=n-k+1}^{n} C_{i}^{n-k} / i^{2}$ and $v_{k l}=2 \sum_{i=n-k+1}^{n}$ $\sum_{j=n-l+1}^{n} C_{i}^{n-k} C_{j}^{n-l} / j^{2}$. 
Example 3. Let $B$ denote the right-hand side of (22). Combining the Chebychev inequality with (22) we obtain the following confidence interval $\left[L-\left(\left(\operatorname{Var} L^{\prime}+B\right) / \alpha\right)^{1 / 2}, L+\left(\left(\operatorname{Var} L^{\prime}+B\right) / \alpha\right)^{1 / 2}\right]$ for the expected value of $L$-statistic from dependent observations with confidence coefficient greater than or equal to $1-\alpha$.

Acknowledgements: The authors would like to thank the referees for their valuable comments which led to improvements in the paper. The research was supported by the Polish Ministry of Science and Higher Education Grant no. N N111 431337.

\section{References}

[1] Acerbi, C. (2002) Spectral measures of risk: A coherent representation of subjective risk aversion. J. Banking Finance 26, 1505-1518.

[2] Arnold, B.C., Groeneveld, R.A. (1979) Bounds on expectations of linear systematic statistics based on dependent samples. Ann. Statist. 7, 220223.

[3] Balakrishnan, N., Lai, C.D. (2009) Continuous Bivariate Distributions. 2ed. Springer

[4] Birkel, T. (1992) Laws of large numbers under dependence assumptions. Statist. Probab. Lett. 7, 17-19.

[5] Blom, G., Holst, L., Sandell, D. (1994) Problems and Snapshots from the World of Probability. Springer, New York.

[6] Darkiewicz, G., Dhane, J., Goovaerts, M. (2005) Risk measures and dependencies of risks. Braz. J. Probab. Stat. 19, 155-178.

[7] David, H. A., Nagaraja, H. N. (2003) Order Statistics. Wiley, Hoboken.

[8] Davydov, Y., Khoshnevisian, D., Shi, Z., Zitikis, R. (2007) Convex rearrangements, generalized Lorenz curves, and Gaussian data. J. Statist. Plann. Inference 137, 915-934.

[9] Denuit, M. Dhane, J., Ribas, C. (2001) Does positive dependence between individual risks increase stop-loss premiums? Insurance Math. Econom. 28, 305-308. 
[10] Doukhan, P. (1994) Mixing: Properties and Examples. Springer, New York.

[11] Dowd, K., Cotter, J., Sorwar, G. (2008) Spectral Risk Measures: Properties and Limitations. J. Finan. Serv. Res. 34, 61-75.

[12] Goroncy, A., Rychlik, T. (2008) Lower bounds on expectations of positive $L$-statistics from without-replacement models. J. Statist. Plann. Inference 138, 3647-3659.

[13] Greselin, F., Puri, M. L., Zitikis, R. (2009) L-functions, processes, and statistics in measuring economic inequality and actuarial risk. Statist. Interface 2, 227-245.

[14] Kaluszka, M., Okolewski, A. (2001) An extension of the Erdős-NeveuRényi theorem with applications to order statistics. Statist. Probab. Lett. 52, 181-186.

[15] Kaluszka, M., Okolewski, A. (2005) Bounds for L-statistics from weakly dependent samples of random length. Comm. Statist. Theory Methods 34, 1899-1910.

[16] Kremer, E. (1998) Largest claims reinsurance premiums under possible claims dependence. ASTIN Bulletin 28, 257-267.

[17] Matuła, P. (2005) On some families of AQSI random variables and related strong law of large numbers. Applied Mathematics E-Notes 5, 3135 .

[18] Rychlik, T. (1998) Bounds on expectations of L-estimates, in: Order statistics: Theory and Methods, (N. Balakrishnan and C.R. Rao, eds.), Handbook of Statistics, Vol 16, North-Holland, Amsterdam, 105-145.

[19] Rychlik, T. (2001) Projecting Statistical Functionals Springer, New York.

[20] Serfling, R. J. (1980) Approximation Theorems of Mathematical Statistics. Wiley, New York.

[21] Szekli, R. (1995) Stochastic Ordering and Dependence in Applied Probability. Springer, New York. 
[22] Wang, S. (1996) Premium calculation by transforming the layer premium density. Astin Bull. 16, 71-92. 